\title{
Material zur spanischen Streikbewegung der letzten Jahre
}

\author{
Pedro Garcia Lopez
}

Am 2. 3. 1974 wurde in Barcelona Salvador Puig Antich und in Tarragona der Pole Heinz Chez von der spanischen Exekutive ermordet. Aus der ganzen Welt trafen vor der Ermordung Gnadengesuche bei Franco ein; es gab zahilose Demonstrationen und Solidaritätsaktionen in vielen Ländern, besonders in Frankreich, aber auch in Italien sowohl vor als nach den Ermordungen. In Deutschland fanden ebenfalls in vielen Städten Demonstrationen statt.

Bei diesen Verbrechen der Militärjustiz, ebenso wie angesichts des Prozesses gegen zehn Arbeiterführer am 20.12.1973 fragt man sich: Wie stark kann die Repression noch wachsen und auf welcher Grundlage? Gibt es die von bürgerlichen Blättern beschworene ,Liberalisierung" wirklich? Was sind eigentlich die Bedingungen des Klassenkampfes in Spanien, wie stark ist die Arbeiterbewegung, welche politischen Gruppen sind in ihr vorherrschend?

Selbstverständlich können wir in diesem Artikel nicht viel mehr tun, als diese Fragen anzuschneiden und am Beispiel eines der täglichen Arbeitskonflikte ein wenig Material für ihre Beantwortung zu liefern. Dazu werden Erklärungen zum institutionellen Rahmen gegeben, dem sich die spanische Arbeiterklasse konfrontiert sieht, sowie zur staatlichen vertikalen Zwangs, gewerkschaft", zum Arbeitsrecht und zu den spezifischen spanischen Repressionsmechanismen. Damit der im folgenden dargestellte Konflikt nicht als isolierter erscheint, ist es notwendig, einen kurzen Überblick über die heftigsten Streikbewegungen der letzten Jahre zu geben:

Im Februar 1969 streikten etwa 15000 baskische Arbeiter für höhere Löhne; im Januar 1970 lag das gesamte Minengebiet in Asturien lahm. Die Ursache für diesen Arbeitskampf liegt in den schlechten Arbeitsbedingungen und der hohen Unfall- und Todesrate des Bergbaus. Wegen der niedrigen Löhne und fehlender fester Arbeitsverträge fanden im März 1970 große Streiks in der Bauwirtschaft Sevillas statt, wobei zweitausend Arbeiter entlassen wurden. Im Juni traten 24000 Bauarbeiter wiederum in den Streik, und es kam zu heftigen Auseinandersetzungen mit der Polizei. Im Juli wurden bei einer Demonstration anläßlich neuer Kollektivabkommen für die Bauwirtschaft in Granada drei Arbeiter von der Polizei erschossen, worauf im ganzen Land eine Welle von Proteststreiks aufflammte. Im Dezember fand der Burgos-Prozeß gegen ETA-Mitglieder statt, der mit der bisher größten Streikwelle in allen Regionen beantwortet wurde. Im Vergleich zu 1969 haben sich 1970 die Zahlen der Arbeitsniederlegungen verdoppelt und die politischen Solidaritätsstreiks übersteigen bereits der Zahl und ihrem Gewicht nach die Arbeitskämpfe um Lohnerhöhungen und verbesserte Arbeitsbedingungen. 1971 wurde im Baskenland im Zusammenhang mit dem Burgos-Prozeß und der starken Streikbewegung für sechs Monate der Ausnahmezustand verhängt. In Madrid begann ein Sireik des Ärzte- und Plegepersonals, der sich schnell über das ganze Land ausbreitete. Neben höheren Löhnen und besseren Arbeitsbedingungen zielte eine 
der wichtigsten Forderungen auf eine Neustrukturierung des unzulänglichen Gesundheitswesens. Im Verlauf des großsen Bauarbeiterstreiks im Sommer 1971 in Madrid, an dem etwa 40000 Arbeiter teilnahmen, ermorde te die Polizei den Arbeiter Pedro Patiño. Im Oktober bese tzten große Teile der knapp 30000 SEAT-Arbeiter während eines Arbeitskampfes ihre Fabriken. Der Anlaß war die Forderung nach Wiedereinstellung von bei einem vorhergehenden Konflikt entlassenen Arbeitern. Es kam zu heftigen Auseinanderse tzungen mit der Polizei und ein weiterer Arbeiter wurde e $e_{1}$ schossen. Daraufhin fanden in Barcelona und weiteren Gegenden der katalanischen Industrieregion Solidaritätsstreiks statt. Im November begann eine neue Streikwelle in Asturien. Es beteiligten sich ungefähr 27000 Arbeiter, vorwiegend Kumpel. Im März 1972 streikten die Arbeiter der Werften von El Ferrol in Galizien, das als Geburtsort Francos, "El Ferrol del Caudillo" heifst (,El Ferrol des Führers ${ }^{66}$ ). Der Streik dehnte sich schnell auf die ganze Stadt aus, auf die die Kanonen der Kriegsschiffe im Hafen gerichtet waren. Die Polizei ermordete wieder drei Arbeiter.

In El Ferrol, später in Vigo und Pamplona, deutet sich eine neue Phase der Klassenkämpfe in Spanien an. Die Streikbewegung beschränkt sich nicht mehr nur auf-die Fabriken, sondern es beginnen ganze Städte am Arbeitskampf teilzunehmen, in Pamplona sogar weite Teile der Provinz. Der Widerhall der Kämpfe von El Ferrol war in ganz Spanien zu spüren. Hunderte von Solidaritätskundgebungen und -streiks fanden statt.

Im September 1972 traten die Arbeiter von Citroễn in Vigo (Galizien) aufgrund von Lohnforderungen in den Streik, der binnen kurzer Zeit fast alle Betriebe

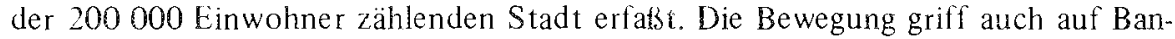
ken, Versicherungen und sogar den Einzelhandel über. In der Stadt wurden Barrikaden gebaut, und es gelang den Einwohnern für einen Tag, die Polizei von den Straßen in die Kasernen zu vertreiben. Am nächsten Tag wurde die Stadt vom Militär besetzt. Zur Unterstützung der Kämpfe in Vigo entflammten auch in El Ferrol wieder zahlreiche Streiks und zugleich entstand eine starke Solidaritätsbewegung in fast allen anderen Provinzen.

Im April 1973 wurde in Barcelona ein weiterer Arbeiter bei einer Demonstration für höhere Löhne und feste Arbeitsverträge in der Bauwirtschaft erschossen. Die zahlreichen Morde an Arbeitern tragen sehr zur Verschärfung der Klassenauseinandersetzung bei, die häufig durch Polizei- und Militäreinsatz beendet werden. In den Flugblättern, die bei Arbeitskonflikten zirkulieren, wird durch die namentliche Aufzählung der erschossenen Arbeiter immer wieder der Zusammenhang von ökonomischen Kämpfen und der Mörderdiktatur hergestellt.

Die vom Arbeitsgericht als berechtigt bezeichnete Forderung der Automobilarbeiter von Pamplona nach einer bestimmten Prämie, die das Unternehmen nicht zu zahlen bereit war, wurce zum Anlaß für die großen Kämpfe im Juni 1973. Den Streiks in der Automobilindustrie schlossen sich in hohem Maße Banken, Versicherungen, das Transportwesen und Bauern an. Die Kämpfe in Pamplona dehnten sich schnell über große Teile Navarras aus. Rings um Pamplona sperrten Busfahrer und Bauern die Straßen, um das Anrücken des Militärs aufzuhalten. Im Sommer 1973 fand in Madrid wieder ein großer Bauarbeiterstreik statt, der durch äußerst brutales Vorgehen der Polizei zerschlagen wurde. Von Oktober bis Dezember streikten wie- 
derum die Kumpel in fast allen Minen Asturiens mit dem Hauptziel, eine Verkirzung der Arbeitszeit durchzusetzen, Das Jahr 1974 begann mit dem Streik bei Standard Electrica in Madrid, bei einem der größten spanischen Unternelmen, auf den wir jetzt etwas näher eingehen werden.

Zur Enatehung des Arbeitskampfes bei Standard Electrica S. A.

Vom 26. 1. bis zum 9.2.1974 befand sich die Belegschafi der spanischen Tochtergesellschaft des multinationalen US-amerikanischen ITT-Ronzens, Standard Electrica S. A. weitgehend geschlossen in einem Vollstreik.

Standard gehort zu 70\% ausländischen Aktionären, allein $60 \%$ der Anteile sind im Besitz von ITT, dessen Konzembudget größer als der Haushalt des spanischen Staates ist, nämlich über $400 \mathrm{Mrd}$. Ptas., das sind etwa $20 \mathrm{Mrd}$. DM; (1 Pesete $=0,05$ DM). Der Konzern beschäftigt über 375000 Arbeiter in 70 Län dem.

In Spanien ist Standard mit einer Belegschaft von über 19000 der Beschäftig. tenzahl nach hinter SEAT (spanische FIAT) das zweitgrobte Untemelmen, dem Umsatz nach im Jahre 1973 an neunter Stelle, der Gewinnsituation nach an erster.

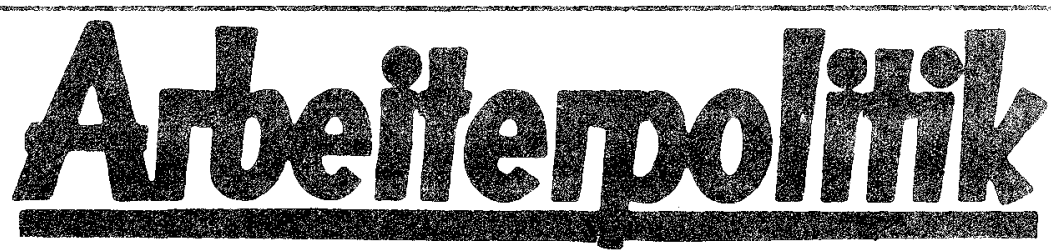

Das seit längerem angekündigte Reprint der Zeitschrift, Arbeiterpolitik" wird aufgrund umfangreicherer editorischer Vorarbeiten erst im Spätsommer 1974 zu erscheinen beginnen. Hiermit sind folgende Änderungen verbunden:

1. Wegen des wahrscheinlich größeren Umfangs der Bégleittexte und -materialien des einzelnen Bandes und wegen der Kostenentwicklung bei der Herstellung wird der Subskriptionspreis wie der Endpreis vermutlich leicht angehoben werden müssen. $16 a$. 16,00 DM bzw. ca. 19,00 DM)

2. Der Gesamtumfang des Reprints wird vermutlich beträchtlich mehr als die bisher angegebenen 3300 Seiten sein, so daB das Reprint eventuell in 13 statt 12 Bänden erscheint.

3. Wegen der Verschiebung des Erscheinungszeitraumes wird die Subskriptionsfrist moch einmal varlängert bis zem 30. Okto. ber 1974.

Bitte in den Buchhandlungen mit sozialistischer Literatur subskri. bieren oder direkt beim Verlag:

Politladen 852 Erlangen Postrach 2849 
Erreicht z. B. SEAT „nur“ 765 Mio. Ptas. (38 Mio. DM) Gewinn, so Standard mit weniger als der Hälfte des Umsatzes über 1,2 Mrd. Ptas. (60 Mio. DM). Das Verhältnis der von Standard verausgabten Löhne zu den ausgewiesenen Gewinnen hat sich zugunsten letzterer wie folgt verändert (dabei ist die Zunahme der Beschäftigtenzahl mit zu berücksichtigen. $(1966=100)$ :

$\begin{array}{ccccc} & \text { Umsatz } & \text { Gewinne } & \text { Löhne/Gehälter } & \text { Beschäftigte } \\ 1966 & 100 & 100 & 100 & 14.644 \\ 1967 & 116 & 173 & 110 & 14.167 \\ 1968 & 120 & 197 & 118 & 13.914 \\ 1969 & 150 & 246 & 140 & 15.026 \\ 1970 & 185 & 333 & 171 & 16.394 \\ 1971 & 235 & 410 & 212 & 18.075 \\ 1972 & 280 & 535 & 223 & 18.800 \\ 1973 & 342 & 668 & 245 & 19.226\end{array}$

Umsatz, Gewinne und Löhne/Gehälter in Mio. Ptas. (1)

ITT unterhält in Spanien mehre re Produktionszentren: Madrid, Santander und Toledo. In Madrid befinden sich zwei Fabriken mit jeweils etwa 6000 Beschäftigten, also insgesamt 12000 , ein Forschungs- und Rechenzentrum mit etwa 500 und Verwaltung und Übriges mit etwa 1400 Beschäftigten. In Toledo sind es 1600 , in Santander 1500 Beschäftigte. ÜUer das ganze Land verteilt arbeiten für ITT, die noch immer das Monopol zur Herstellung der spanischen Telephonapparaturen innehat, weitere 2000 A rbeiter an Installationen.

Von den großen Unternehmen in der Metallbranche der Provinz Madrid zahlt Standard die niedrigsten Löhne. $75 \%$ der Belegschaft sind Spezialisten und erhalten monatlich an grantierten Lohn/Gehalt 9513 Ptas. (knapp 480 DM). Dazu kommen betriebliche Zulagen, die als persönliche Vergünstigungen vertraglich nicht abgesichert sind. Der Durchschnitt des effektiv gezahlten Lohnes ist aufgrund dieser individuell stark differenzierten Lohnstruktur schwer zu ermitteln; vermutlich liegt er jedoch noch unter 10000 Ptas. (500 DM) (2).

Der letzte „Tarifvertrag“ (Firmen,,tarifvertrag" mit jeweils zweijähriger Laufzeit) wurde im September 1973 gekündigt und lief am 31.12. 1973 aus. Die Verhandlungen begannen erst am 15.1., nachdem die Arbeiter dieses Schlüsselbetriebes der zentralspanischen Metallbranche bereits monatelang ausgedehnte Umfragen durchgeführt hatten und das Erge,bnis in Form eines Vertragsentwurfs dem „Betriebsrat" vorlegten. Dieser Entwurf, dem über 5000 Unterschriften beigefügt waren, spiegelte sehr detailliert die Meinung des größten Teils der Arbeiter wider. Von den 21 Forderungen waren die wichtigsten:

-- 500 Ptas. (ca. 25 DM) täglicher Mindestlohn und 5000 Ptas, garantierte moratliche Mindestlohnerhöhung einheitlich für alle Kategorien.

- $\quad$ 40-Stunden-Woche (bisher 43,5)

- $\quad 30$ Tage Jahresurlaub (bisher 20)

1) Cambio 16, Nr. 117, 11.2.74, \$. 43

2) Cambio 16, Nr. 118, 18. 2. 74, S. 15 
- Lohnerhöhung nach Länge der Betriebszugehörigkeit alle drei, statt bisher fünf Jahre.

- $\quad 100 \%$ des Effektivlohns als Ruhegeld mit 60 Jahren

- Die $12 \%$-Steuer für Prođuktionsausfälle oder -verminderung, die die Arbeiter aufzubringen haben, zu Lasten des Betriebes.

- Beteiligung einer von den Arbeitern demokratisch gewählten Kommission an den Verhandlungen.

- Versammlangsrecht etc.

Der „Betriebsrat" von Standard setzt sich aus 16 von den Arbeitern nicht direkt gewählten Personen zusammen. Jedes Betriebszentrum stellt 4 Delegierte, so Madrid mit etwa 14000 ebenso 4 wie Santander und Toledo mit jeweils etwa 1500 , sowie der Installationsbereich. Zwei Drittel der Mitglieder des „Betriebsrats" vertreten Unternehmerinteressen. Die Verhandlungskommission bildet sich aus diesen 16 Leuten, sowie 2 Sekretären und einem Vertreter der „Beratungskommission“ des vertikalen Syndikats. Das Unternehmen stellt ebenfalls 16 Verhandlungsperso. nen.

Anfangs tritt der ,BR“ zur Beratung des Entwurfs der Arbeiter in Madrid zusammen, dann, als die Arbeiter deutlich ihre Interessen und ihre Verbitterung über die lange Hinauszögerung des Verhandlungsbeginns in Versammlungen, Pe titionen usw. bekräftigen, siedelt er nach Santander über, wo der Druck der Arbeiter geringer ist. Die politischen Forderungen der Arbeiter werden ausnahmslos gestrichen, wie Versammlungsfreiheit und Streikrecht, ebenso die Übertragung der $12 \%$-Steuer auf das Unternehmen, der Zugehörigkeitszuschlag alle drei Jahre, der Urlaub wird auf 27 Tage verkürzt usw. Immerhin bleibt aber durch die Entschiedenheit der Arbeiter der wesentliche Teil der Lohnforderungen bestehen und die Arbeiter beschließen, diesen verstümmelten Entwurf dennoch in den Verhandlungen zu unterstuitzen.

Zum Verständnis des Ablaufs des Arbeitskampfes bei Standard erscheint es uns sinnvoll, zuvor einige Unterschiede zu verdeutlichen, die zwischen Tarifverhandlungen in den westlichen kapitalistischen Ländern und dem System der spanischen „Kollektivabkommen" bestehen. Selbst der Begriff „Kollektivabkommen" ist in einem strengen Sinne falsch, da er zweiseitige Verhandlungen gegenerischer Parteien vortäuscht und Assoziationen an einen Tarifvertrag weckt. Als Verhandlungspartner sitzen sich in Spanien das Kapital und Vertreter der staatlichen Einheits, gewerkschaften $^{\text {" gegenüber. }}$

Aufgrund großer Verschiedenheit von den in Westeuropa gebräuchlichen Formen der Lohnfestlegungen ist dazu eine Erläuterung des spanischen Lohnsystems sinnvoll.

\section{Kapitalakkumulation und Lohndiktat}

Vom Ende des Bürgerkrieges (1939) an diktierte der faschistische Staat vollständig die Löhne. Mit dem Ziel einer Vollbeschäftigungspolitik setzte er sie äußerst niedrig fest und stellte zugleich ein allgemeines Entlassungsverbot auf. Bis in die Mitte der 
funziger Sahe hell das Regine an einer autarkistischen Wirtschaftspolike fest, die z. T. eine Antwor auf die intematonale Blockade Spaniens als einzigem faschistischem Lnd nach dem Ii. Weltkreg darstellte. In der Zeit des Kalten Krieges begannen die Vercingien Stadten als erste im Jahre 1951, Wirtschafts- und Militarhilfe an Spanien zu leisten. Ein anhaltender wirtschafticher Aufschwung fand aber auch in den funfiger Jahren nicht statt, und etwa Mitte des Jahrzehnts war die Wirtschaft weirgehend zugnude gerichtet: Die Produktiviat war äuserst gering, was neben den Fogen kes Krieges groknteils der staatichen lohn und Beschärigungsolitik geschudet war. Das Evidasungsveroot enerseits und de niedrigen lobne anderer. seits venthderen ene Rationalisienng in den Betrieben. Durch de geringe ${ }^{3}$ roduk. tivität und den technologischen Ruckstand der spanischen Wirtschaft waren thre Produkte intemational nicht konkumenzahig, hinzu komn, dab de hauptsächlich aus diesen Pedingungen resultierende autakistishe Wrtschaftspolik sich schnell in eln Dognáa vercsigte und mogliche Initativen fur eine Offnung nach Europa ióologisch abschit. Die Peseta war nicht konvertibel. Un hnvestitionen zu in-

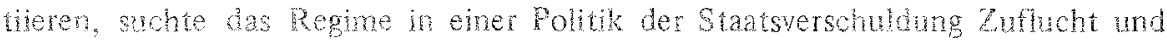
trieb die Gnlationsrate in die Hohe. Der gleichzeitge Lohn und Preisdirgismus hielt die Pressentwicking nicht auf (3). Zwar funktionierte das Lohndiktat, nicht aber der Freisstop. Dis Arbeiterklasse reagierte auf diese Verschechterung der Le. bensbedingmgen mit groken Streikbewegungen ab 1951 .

Als einzige Ouelle der Akkumulation verblieb dem Staat ene Niedriglohnpolithk, die er ber absolutes Lohndiktat durchseate. Zugleich verfolgte er hermit auf Kosten der Arbeiterklasse das Ziel, die inflationäre Entwicklung zu bremsen. Diese Politik war neben dem Entlassungsverbot noch in einem zweiten Sinne Beschäftigungspohik: Durch die geringe Lohnhohe solte den Untemehmen ermoglicht werden, alie beim gegeberen, niedrigen Produktivitätsstand beschäftigten Arbeiter im Betrieb zu halen. Das Resultat war en Stagnieren der Produktiviätsziffen. Die Zentralisation des Kapitals wurde damit verhindert und die grofe Masse der Kleinbetriebe, die die soziale Hauptstitze des Regimes darstellt, konnte sich am Leben halten. Als dann allnähich, vor allem aufgund der US-Kredite, gegen Mite der finfziger Jahre die Nachfrage anstieg, löste sie keine Rationalisierungsmaßnahmen aus, sondern schug sich fast vollsündig in Form erhöher Preise neder (4)

Das starke Ansteigen der Geldentwertung in den Jahren 1954/55 fuhrte zu einer gewissen Lockerung staaticher Regulienngen. In diesem Zusammenhang ist besonders die Auhebung des staatlichen Lohndiktats im Jahre 1956 von Bedeutung. Zwar legte der Stat immer noch die Löhne fest, sie konnten aber von nun an ohne staatliche Genehmigung uberschritten werden. Ursache dieser newen Regelung sind die ab 1951 wieder zunehmenden Arbeitskämpfe, die sich grobenteils gegen die staatiche Niedriglohnpolitik richten. Zugleich lag die Durchlässigkeit des Lohndiktats im Interesse dexjenigen Kapitale, die hohere als die staatlich festgelegten Löne zahlen konnten. Das waren v. a. größere Untemehmen mit den me isten Arbeitskon-

3) vgi. Internationales Arbeitsant (OIT): La siuación laboral y sindical en España, Genf 1969 , Ziffer 106

4) vgl. Ulrich Brösse, Wirtschaftsordnung und Arbeitsrecht in Spanien, Stuttgarî 1965, S. 46 
flkten aup sich verenigten. Diese Kapitale waren noht nu daran interessit, ine Produrton relativ storungstrei aufrechzuehalen, sondem wollen gleicheitg einer Politisicung und Organisierung der Arbeiter durch manifeste Abeiksonnkte aus dem Wege gehen, zumal jener vom Enzelkapital as geschenen , nicht notwen.

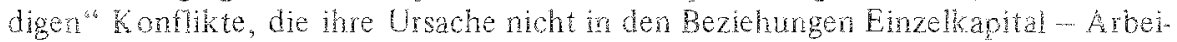
ter haben, sondem in der stathch Rom der Lohfestsetzung, wodurch die Lohn konhikte von Anfang an enen iber den winzelbetrieb hmawsweisenden offen politschen Charaker hatten.

Die Unterehmen vehandelten von mun an informell und ab abueronzel lem Wege mit den Arbeiten uber Lohnehohungen. Wur den Stat brachte das die Gefahr mit sich, die Durchsetzungsmöglichkeiten seiner lohi und beschartgungspolth zunehmend zu verleren. 1958 wurde das Gesetz boer Kollektiabkonmen erlassen, womit der Staâ senen Einhub zunckzugewhen sucht. Santiche Varhandungen bber Abeitsbedingungen und Lohne whden dami auf das statiche Einheitssydika beschänkt. Das Syndikat indient die Verhandungen, Thr sie durch, erklär sie für gescheiten usw. Das bedeutet fur die Arbeiter, dak sie the Forderungen atur noch an ine offiellen, Ventreter im syndicat weitergeben dirfen.

Dieses Geselz ist democh nicht als Marnahme des Staates gegen das Mapla mibzuverstehen, denn fü das Kaptal war die alte Regelung des lohndavatswegen des Wiorstandes der Arbeiter politisch zu gefämbh geworen. Mit den neven Gesctz ehofite man sich ene hregration der Abeiter und ene stakere ldemtifika. tion mit den von ihen eigenen ,Vertreten" ausgehandelten Arbeitsbedingugen, ein gröberes Mas an Zustimmung zu den beschlossenen Lohnerhohungen und danit auch das Einhalten der Laufzeiten.

Die ab 1956 neu an die Mach gekommenen Opus Den Techokraten, die ene wirtshafthohe Offing Spaniens besonders nach Europa anstrebon, visierten eine

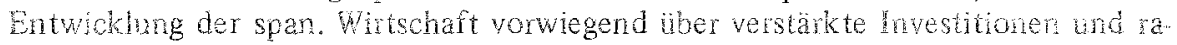
sches Produktiviätswachstum an, was sich an den Soll-zahlen der jevells verjahrigen Entwicklungsplane ablesen läß Um diese Ziele zu erreichen, war eme Verandenug der Abeitsbedingungen notwendig. Das Gesetz uber die Kollekivabommen stellt den Versuch dar, die zu erwartenden Reaktionen der Arbeiter auf die Ver. schlechterung iher Arbeitsbedingungen auf dem Wege berieblicher Lohnverhandlungen zeithch und regional $z u$ ischeren. We Arbeiterklase hatte in den letzen Jahren vor detu Gesetz rasch an Kamphraft gewonnen und ihe Kampfe begannen sich immer stäker $z$ veralgemeinem. Durch das neve Gesetz konnten die nun schon emmal vohandenen Konfliktzonen zugleich besser kontrolliert werden (5).

Für den Stat war die Wiedererangma emer Basis fur seine Lohnolitik anch nur enes der Ziele des Gesetzes: Mzwischen hatte sich die Ungangbakeit des autarkistischen Weges hinreichend bewiesen und eine Nevorientierung der Wirt schaftspolitik war unawweichlich. Das war die Bedingung fur den Aufstieg des Oprs Dei in die Ministersessel. Sie entwickelten erstmalig mittelristige staatiche Wirtschafsplane. Ghe neue wirtschaftspolitische Orientierung schlug sich in der 1 ohn-

5) Vgl. El conticto obreco en España 1960-1970, 2 Bände, Irstituto de Estudios Laborales, Barcelona 1972, unveröfenticht. Bd. II, S. 326 
politik nieder. So sollen Lohnerhöhungen nur gewährt werden, wenn sie mit Produktivitätssteigerungen einhergehen. Im wesentlichen verfolgte das Gesetz iiber die Kollektivabkommen die Absicht, die Wachstumsraten der'Produktivkräfte zu erhöhen, wobei gleichzeitig die Bindung der Lohnsteigerungsraten an die Wachstumsraten der Produktivität als stabilitätsgerechte Politik gepriesen wurde und wird. Mit der Wachstumsrate der Produktivität ist nicht die des jeweiligen Einzelkapitals gemeint, sondem die des nationalen durchschnittlichen Wachstums, wodurch den kampfstärksten Sektoren der spanischen Wirtschaft Lohnsteigerungen zugemutet werden, die immer weit unter dem Produktivitätswachstum ihres Sektors liegen. Daß daraus weitere Anlässe zu Lohnkämpfen bz'w. deren Verschärfung entstehen, ist einleuchtend, und die entsprechenden Kapitale geraten durch den Druck ihrer Arbeiter oftmals in Gegensatz zur staatlichen Lohnpolitik.

Obgleich die Rate des Produktivitätswachstums mit Lohnsteigerungen in Wirklichkeit nicht in Zusammenhang steht, ist es interessant, die spanische Lohnentwicklung an dem postulierten Ziel zu messen, nämlich, die Löhne der Produktivitätssteigerung anzupassen. Im Zeitraum 1964-1970 bleibt in der Industrie die Rate der Lohnsteigerungen mit Ausnahme von 1966 und 1967 ständig unter den durchschnittlichen Produktivitätszuwächsen, 1969 erreichte aufgrund der Lohneinfrierung sogar nicht einmal die Nominallohnerhöhung diese Zuwachsrate. Dennoch hatte gerade die Industrie die höchsten Zuwachsraten der Produktivität zu verzeichnen (6). Im tertiären Sektor überschreiten nur 1970 die Lohnwachstumsraten die Wachstumsraten der Produktivität Dennoch werden vom Regime die häufigen Lohnstops und Einfrierungen damit begründe $t$, daß die zu starken Lohnerhöhungen den inflationären Prozeß bedingten. Von 196-1971 blieb der Anteil der Löhne und Gehälter am Nationaleinkommen konstant, obgleich die Lohnbevölkerung zunahm.

Mit dem Gesetz über Kollektivabkommen hat der Staat die Möglichkeit für eine etwas flexiblere Lohnanpassung geschaffen, keineswegs aber hat er Tarifautonomie hergestellt. Er setzt jährlich Höchstgrenzen für Lohnsteigerungen fest, wenn er nicht sogar wie 1968 vollständigen Lohnstop verfügt. Diese Höchsigrenze darf die Summe derjährlichen Rate des Produktivwachstums und der Preissteigerungsrate nicht überschreiten und war z. B. 1973 14\% für Verträge mit einer Laufzeit von einem Jahr und ist $21 \%$ für Zweijahresverträge (1973/74), die am häufigsten abgeschlossen werden. Zugleich lag 1973 der Anstieg der Lebenshaltungskosten nach Angaben des Nationalen Statistischen Instituts (INE) schon bei $142 \%$ (7). In den

6) vgl. Quijada/Rachdorf: Zur soziookonomischen Entwicklung Spaniens, in: SoPo 23, S. 9; sowie Cruz/Serrano: Economía laboral española: Negociación colectiva y visión global de 1972, in: Muñoz/Roldán/Garcia Delgado: La economía española 1972, Madrid 1973, S. $160 \mathrm{f}$.

7) Die Methode der Erstellung eines ,Warenkorbes“ als Grundlage der Preisstatistik ist in Spanien eine andere als in der BRD. Es ist aber nicht bekannt, wie seine Elemente zusammengesetzt sind. Nach Berechnungen einer katholischen Unternehmerzeitschrift (Documentación para dirigentes, Dezember 1973) belief sich die Steigerung der Lebenshaltungskosten in Barcelona 1973 auf $22 \%$. Fast alle anderen Berechnungen seitens privater Institute liegen weit über den Angaben des INE, dessen Daten aber deshalb von größter Bedeutung sind, weil sie zugleich die Hauptgrundlage der Höchstgrenze für Lohnerhöhungen darstellen. (Vgl. El aumento del coste de la vida y las medidas del gobierno. Documentos informativos del Instituto de Estudios Laborales, Barcelona, März 1974) 
Monaten Januar bis März 1974 sind laut INE die Preise außerordentlich stark gestiegen; im März 2,17\%. In diesem Vierteljahr hat sich die Lebenshaltung be reits um $3,10 \%$ verteuert; im gleichen Zeitraum 1972 um 0,98\%, 1973 um 1,74\%. Normalerweise pflegen in Spanien die Lebenshaltungskosten erst in den Sommermonaten kräftig anzusteigen, was dem Touristenstrom geschuldet ist (8).

Ab 1963 dekretiert der Staat einen generellen Mindestlohn, der nicht unterschristen werden darf. Dieser Mindestlohn ist auf einen achtstündigen Arbeitstag eines Hilfsarbeiters berechnet und betrug bisher (April 1973 bis März 1974) 186 Ptas. (etwa 9,30 DM) und ist zum 1. April 1974 auf 225 Ptas. (knapp 11,00 DM) angehoben worden. Als Effektivlohn spielt der staatliche Mindestlohn keine große Rolle, da er in der Regel überschritten wird. Seine wirkliche Funktion besteht aber darin: Indem der Staat diese Lohnhöhe festsetzt, wirkt er direkt auf die Unternehmerangebote in den Lohnverhandlungen und drückt sie herab. Auf diese Weise entstehen dann die in Spanien üblichen äußerst niedrigen „Basislöhne", welche plus weniger verschiedener Zulagen den ,tariflich" abgesicherten Teil ausmachen und oft nur knapp über den staatlichen Mindestlöhnen liegen. So bleibt großer Raum für eine individuelle Lohnbemessung. Der über den ,Tarif"lohn hinaus gezahlte Lohnteil besteht aus bis zu 90 verschiedenen Arten individueller Zulagen, die sich bisweilen der Höhe des ersteren annähern (9).

Zugleich mit dem Gesetz über Kollektivabkommen von 1958 hat der Staat sich ein Instrument geschaiffen, um sich die Dürchsetzung seiner Lohnpolitik zu sichern: die Praxis der verpflichtenden staatlichen $Z$ wangsrichtlinien (,normas de obligado cumplimiento"). Sie werden immer dann angewandt, wenn es zwischen den „Arbeitervertretern" des Syndikats und den Kapitalvertretern nicht zu einer Einigung kommt. In diesem Falle ,,schlichtet" der Staat, d. h. er oktroyiert eine Lohnregelung, die nie über die von ihm festgelegten Höchstgrenzen hinausgeht. Damit entfernt er sich in der Regel kaum vom Vorschlag des Unternehmens, welches Lohnerhöhungen innerhalb des staatlichen Rahmens anbietet. Außerdem geht es nicht ausschließlich darum, denn neben den Lohnerhöhungen kämpfen die Arbeiter fast immer um eine höhere tarifliche Absicherung ihres Effektiviohns. Die in den Kollektivabkommen erreichten Erhöhungen werden häufig mit den Zulagen verrechnet, so daß der effektiv gezahlte Lohn gleich bleibt. Über die Möglichkeit für das Kapital, so verfahren zu können, entscheidet jeweils die Kampfkraft der Arbeiter. Besonders in kampfstarken Sektoren setzt der Staat seine Lohnpolitik mittels der Zwangsrichtlinien durch, vor allem also in der Metallindustrie, dem Transportwesen, in den extraktiven Industrien, im Bausektor und in der Textilindustrie. Normalerweise sind die staatlich oktroyierten Lohnerhöhungen niedriger als die ausgehandelten (10).

Besonders wegen der seit 1970 schnell zunehmenden Arbeitskonflikte scheint sich folgende Taktik des Kapitals durchzusetzen: Das Unternehmen legt in den Verhandlungen ein Angebot vor, welches meist nur knapp über dem bisherigen Basislohn liegt; so auch im hier dargestellten Konflikt bei Standard Electrica. Wenn

8) Daten nach: „Informaciones", 19.4.1974

9) s. Cruz/Serrano, a. a. O., S. 154

10) Ebd., S. 184 
die ,Arbeitervertreter", d. h. das Syndikat, das Angebot nicht akzeptieren, ziehen sich die Kapitalvertreter mit dem Hinweis zurück, es sei kein Abkommen zustande gekommen. Daraufhin schaltet sich automatisch der Staat ein, der im besten Falle seine Höchstgrenzen oktroyiert, die das Kapital fast immer mit den Zulagen verrechnen könnte, wenn der Druck der Arbeiter es zuließe. Diese Praxis des Lohndiktats, in welcher der Staat augenfallig als Bütel des Kapitals auftritt, trägt sehr dazu bei, dafs die Lohnkonflikte an Schärfe zunehmen und von Anfang an als politische Kämpfe gefuhrt werden. Diese Zunahme zeigt sich auch daran, daß inzwischen der Teil und Vollstreik alle anderen Formen des Arbeitskampfes, whe: rotierende Ar. beitsnicderlegungen, langsam arbeiten, Petitionen usw, verdrängl hat (11).

\section{Biedriglohngolitik und politische Repression}

Die staatliche Durchsetzung der Niedriglolmpolitik in Spanien bedient sich noch einer ganzen Reihe anderer Repressionsinstrumente, wovon die wichtigsten das Versamrilungsverbot und das Streikverbot sind.

Obgleich täglich im ganzen Land Streiks stattfinden, ignoriert die Rechtsprechung jegliche Art von Kollektivkonflikten. Streikende Arbeiter werden individuell nach dem Strafrecht verurteilt. Der entsprechende Artikel 222 lautet: „Es werden des Aufruhrs für schuldig befunden: ... 2. Arbeitgeber und Arbeiter, welche mit dern Ziel, die staatliche Sicherheit anzugreifen, seinem Ansehen Schaden zufïgen, seine normale Tätigkeit stören oder in schwerwiegender Weise die nationale Produktion unterbrechen oder die Regehnäßigkeit der Arbeit beeinträchtigen." Dieser Text stammt von 1965 und stellt eine Modifikation des urspringlichen Artikels von 1944 dar, welcher noch stark vom Gesetz uber Staatssicherheit (1941) und dem über militärische Rebellion (1943) geprägt war. Bis 1965 hatte es ganz schlicht geheiben: „Es werden des Aufruhrs für schuldig befunden: ... 3. Die Streiks von Arbeiten" "(12). Die Streiks haben ab Anfang der sechziger Jahre derart zugenommen, daßs eine so drakonische Rechtsprechung wie in den vierziger und fünfziger Jahren immer breitere Solidarisierung und Widerstände hervorrief. Vielleicht spielte auch der internationale Druck für die Modifikation des Artikels eine gewisse Rolle. Seit 1958 ist Spanien Mitglied in der OECD (OEEC), im Internationalen Währungsfonds (IWF) Entwicklungsbank und anderer internationaler Organisationen und stellte 1962 einen Assoziationsantrag an die EWG.

Wegen der beim achtstündigen Arbeitstag oft unter dem Existenzminimum verbleibenden Löhe sind die Arbeiter und häufig auch Angestellte in Banken und Versicherungen, Lehrer usw. zu Überstunden bzw. Nebenberufen gezwungen. Der Höchstarbeitstag beträgt laut Gesetz von 1931 acht Stunden täglich und 8 Std. in der Woche. Für $90 \%$ der unteren Angesteliten und $84 \%$ der Arbeiter traf 1971 offiziell der Höchstarbeitstag zu. Dazu kommen Überstunden, die sich so weit verallgeneinert haben, daß sie inzwischen durchaus als Teil des Normalarbeitstages

i1) $\operatorname{Lbd}, 5.232$

12) Ebd., S. 130 fr; sowie Almansa Pastor: la huelga laboral en España tras la modîicación de ar ticujo 222 del Código Penal 
betrachtet werden. Durchschnittlich arbeitete 1972 der Angestellte 48 Std., der Arbeiter $58 \mathrm{Std}$. Uberstunden werden nur mit $125-150 \%$ vergütet (13). Die sehr weit verbreitete $Z$ weitbeschäftigung ist statistisch so gut wie gar nicht zu erfassen und ist daher in diesen Angaben nicht enthalten. Ein Verfahren wegen Uberschreitung des Höchstarbeitstages wird für das Kapital auch nicht teuer: Die Buße beträgt seit 1931250 Ptas. (etwa 12,50 DM). Ab etwa 1972 ist die 40-Std.-Woche eine der wichtigsten Forderungen der Arbeiterbewegung. Die starke Ausdehnung der Arbeitszeit trägt dazu bei, daß Spanien absolut die gleiche Zahl an Arbeitsunfällen hat wie die BRD und relativ an zweiter Stelle in Europa liegt.

Es wurde angedeutet, daß es nicht nur wie in Westeuropa die ,reinen" Beziehungen von Kapital-A rbeit sind, die die Politisierung und Organisierung der Arbeiter bewirken, sondern auch die besonders repressiven Formen, in denen Gesamtkapitalinteressen durchgesetzt werden. Eine besondere Bedeutung für die Politisierung haben die Zwangsrichtlinien, der ständige Polizeieinsatz und die fehlende Arbeitervertretung.

Die Funktion der polizeilichen und militärischen Repressionsinstrumente hat sich für die spanische Arbeiterklasse in der frankistischen Epoche, vergröbert ausgedrückt, umgekehrt. Durch die Zerschlagung der starken Arbeiterbewegung durch den Bürgerkrieg und vor allem in den Terrorjahren der Weltkriegszeit, in dem Hun-

13) Cruz/Serrano, a. a. O., S. 180

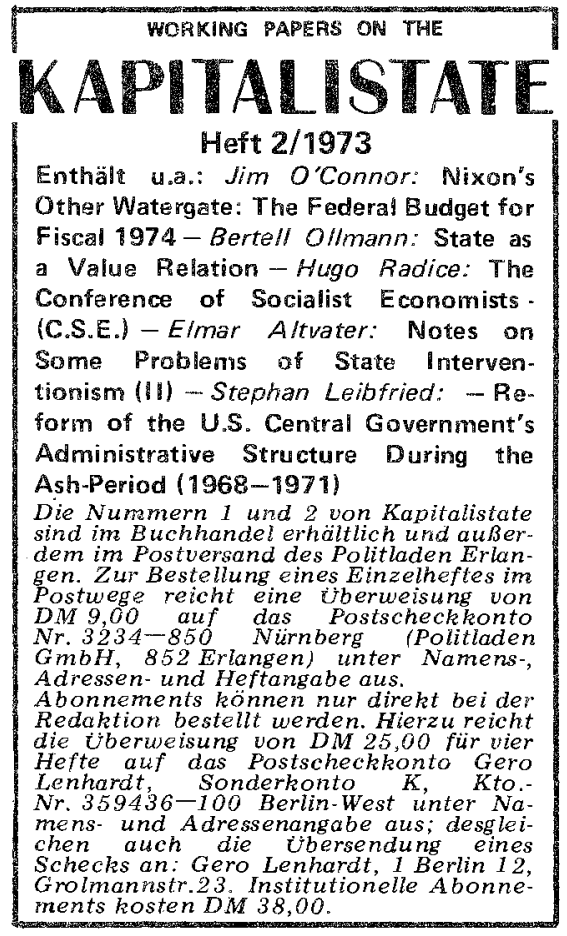

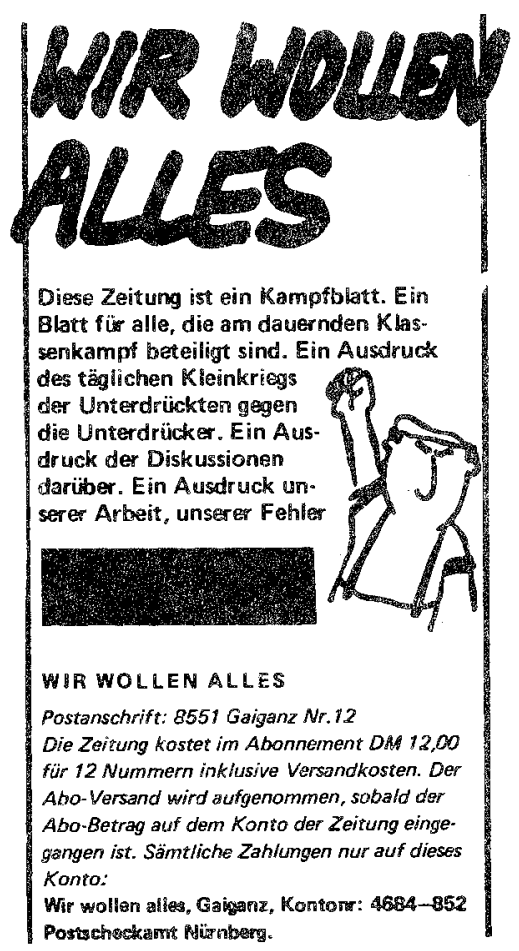


derttausende kämpferischer Arbeiter ermordet wurden (14), war es zunächst tast unmöglich, gegen die staatlichen Repressionsmaßnahmen breiten Widerstand zu leisten. Doch bereits am 1. Mai 1947 fand der erste große Streik statt. Inzwischen hat sich die Arbeiterklasse in Spanien verdoppelt und ist eine der jüngsten Europas. Obgleich die Repressionsmethoden weitgehend die gleichen geblieben sind: Versammlungsverbot, Streikverbot, de jure gleiche Strafen für gleiche „Delikte" wie in den vierziger $\mathbf{J}$ ahren, ist ein wesentliches Kennzeichen der Repression in den letzten Jahren, daß sie zwar noch zur gezielten Unterdrückung in der Lage ist, ihre Einschüchterungsfunktion aber schon weitgehend verloren hat und daher v.a. den allgemeinen Politisierungsprozeß nicht nur nicht mehr aufhalten kann, sondern ihn geradezu mithervorbringt. Das bisher bekannte $\mathrm{Maß}$ an Repression reicht nicht mehr aus, seinen Zweck für das Regime zu erfüllen. Der hohe Grad der Politisierung drïckt sich in der großen Zahl von Solidaritätsstreiks aus, vor allem mit entlassenen und verhafteten Arbeitern. Nach einer Statistik des Arbeitsministeriums war 1970 bereits die Hälfte aller in Spanien durchgeführten Streiks politisch-sozialer Natur, d. h. hauptsächlich Solidaritätsstreiks (15). Eine wesentliche Ursache für diese Entwicklung liegt in der von Anfang an notwendigen Verschmelzung der politischen mit der ökonomischen Seite der Kämpfe.

\section{Das vertikale Zwangssyndikat}

Eines der größten Hindernisse für die Arbeiterbewegung und zugleich ein wichtiger Anlaß ihrer Politisierung und Unterstützung autonomer Organisationen stellt das vertikale Zwangssyndikat dar. Darin sind Kapital und Arbeit zusammengefáß乃t. Seine Funktion besteht nach der Verfassung von 1967 darin, ,die beruflichen und ökonomischen Interessen zur besseren Wahrnehmung der Ziele der nationalen Ge$\mathrm{rr}_{2}$ ?inschaft (zu) kanalisieren." Ideologisch hört sich die Rechtfertigung des vertikalen Syndikats so an: „Indem sich die individuellen wirtschaftlichen Interessen in syndikalistische Formen einordnen, verwandeln sie sich in vertikale Gruppen mit wirtschaftlichem nationalem Interesse. Auf diese Weise stimmt das Individuelle mit dem Nationalen überein und der Gegensatz zwischen Individuum und Staat verschwindet" (16).

Trotz des neuen Gewerkschaftsgesetzes von 1971 hat sich an Struktur und Funktion nichts wesentliches geändert: Noch immer müssen vom Staat eingesetzte höhere Funktionäre jeden von den Arbeitern zu wählenden Vertreter bestätigen und noch immer kann das Syndikat nicht als Vertretung der Arbeiter betrachtet werden. Es findet sich kaum ein Arbeiter, der im Syndikat seine Vertretung sieht

14) In Schätzungen taucht meistens die Zahl 200000 Toter ,durch Francos Repressalien und Gewaltakte" auf; ,,dazu kommen rund 200000 Tote durch Exekution und Tod in Gefangenenlagern in der Zeit zwischen 1939-1943." Hinzu kommt eine Zahl von 350000 Emigranten, von denen 70000 bis 1942 umkamen, vor allem durch die Repressalien der Vichy-Regierung in Frankreich. Siche von Beyme, Vom Faschismus zur Entwicklungsdiktatur-Machtelite und Opposition in Spanien, München 1971, S. 102

15) siehe Cruz/Serrano, a. a. O., S. 230

16) Legaz-Lacambra, zit. nach Brösse, a. a. O., S. 26 
und Studien aus dem Jahre 1965 zeigen, daß selbst $30 \%$ der Unternehmer dieser Meinung sind. Innerhalb der Arbeitnehmersektion des Syndikats selbst sind es sogar über $50 \%(17)$.

Das Syndikat ist aber offensichtlich nicht zu ändern, ohne die Prinzipien des Systems selbst umzuwerfen. Eine Demokratisierung des Syndikats und damit freie Wahlen der Arbeitervertreter oder gar freie Gewerkschaften setzt die Anerkennung des Gegensatzes von Kapital und Arbeit voraus. Die Existenz dieses Gegensatzes zu verneinen ist aber der Kernpunkt der faschistischen Ideologie des spanischen Re. gimes, weiche auf die italienische Carta del Lavoro zurückgeht. Das Syndikat ist die Verkörperung dieser Ideologie. Jede Liberalisierung der Lage der Arbeiterklasse muß sich daher zuerst gegen die syndikalen Prinzipien wenden. Polizei, Militär und die aufgeblähte syndikale Bürokratie, in der die Masse der traditionellen Falangisten sitzt, sind die realen Stützen des gegenwärtigen Regimes und begründen die Unbeweglichkeit des Frankismus, der, um sich zu retten, angesichts zunehmender Klassenkämpfe keine Möglichkeit hat, größere Freiheiten zu gewähren, sondern - so erscheint es gegenwärtig - nur durch zunehmende Repression weiterleben kann.

\section{Der Streik bei Standard Electrica in Madrid im Januar/Februar 1974}

Nach diesen einführenden Erläuterungen wollen wir nun mit der Schilderung des Verlaufs des Arbeitskampfes beginnen:

Die Verhandlungen beginnen am 15. 1. 1974.

Die „Betriebsräte" lehnen eine Teilnahme der von den Arbeitern vorsorglich gewählten Überwachungskommission mit dem Argument ab, sie sei nicht legalisiert worden. Bereits im Oktober 1973 hatten die Arbeiter einen mit 1200 Unterschriften versehenen Brief an das Metallsyndikat mit der Forderung gerichtet, eine Versammlung über ihren Vertragsentwurf abhalten zu können. Weder vor noch während des Arbeitskonfliktes erhielten die Arbeiter eine Antwort darauf. Am gleichen Tag (15. 1.) erfahren die Arbeiter, daß Standard einen Gegenentwurf mit folgendem Angebot vorgelegt hat:

- Lohnerhöhung um 1600 ptas. monatlich für alle Kategorien.

Die weiteren Forderungen zu behandeln lehnt Standard ab, da sie in den Rahmenvertrag gehörten, der bereits im Juli abgeschlossen worden sei. Es gehört zur Strategie des Kapitals, Kollektivabkommen auf Lohnfragen zu beschränken und die Regelung aller anderen Forderungen, wie vor allem die der Arbeitsbedingungen, in den Rahmenvertrag abzuschieben. Die Arbeitsbedingungen werden fern von den Arbeitern ,verhandelt", auf Ebene eines Sektors einer Provinz oder sogar national. Der Einfluß der Arbeiter ist geringer, weil sich ihre Kampfkraft zugleich auf Lohnforderungen richten muß und ihr Kampf gespalten wird. Die stärkere Kampfkraft im Lohnbereich versucht der Staat mit seinen verbindlichen Richtlinien zu brechen.

$\mathrm{Da}$ die vertragliche Absicherung nur einen Teil des Effektivlohns umfaßt, läuft dieser Vorschlag daher auf z. B. 29 ptas. Erhöhung pro Tag für einen Spezialisten hinaus, das sind knapp 1,50 DM, die theoretisch mit den Zulagen auch noch verrechnet werden können.

17) Cruz/Serrano, a. a. O., S. 125 
Am nächsten Tag, dem 16. 1. reagieren die Arbeiter auf diese Unverschämtheit des Betriebes mit Vollstreik. Standard sieht sich gezwungen, das Angebot zu erhöhen. Vorschlag: 49 ptas. Erhöhung pro Tag (knapp 2,50 DM), aber ,um diese wirtschaftichen Verbesserungen gewähren zu können, kann der Vertrag erst am 1. 2. 1974 in Kraft treter!".

Am 17. 1. wird in den beiden großen Madrider Fabriken eine Stunde lang voli gestreikt, und es finden von jetzt an täglich zwei von den comisiones obreras (Arbeiterkommissionen) einberufene Versammlungen statt.

Am 18. 1. wird über Mittag in keinem der Madrider Standard-Betriebe gearbeitet. Die Verwaltung und das Forschungs- und Rechenzentrum, welches zu zwei Dritteln aus sog. höheren Angestellten besteht (Industrie- und Fernmeldeingenieure, Naturwissenschaftler), schließen sich dem Streik an, nachdem sie bereits von Anfang an die Forderungen der Arbeiter unterstützt und übernommen hatten. Auch in Toledo finden am 19.1. (Samstag) und 21.1. (Montag) Streiks statt. Standard hatte die nächste Verhandlungsrunde für den 20. 1. in der Absicht vorgesehen, den Firmenvorschlag durchzubringen. Die Einheit der Arbeiter der verschiedenen Zentren machte diesen Versuch zunichte. Stattdessen beantragte Standard, die Verhandlungen wegen "des anormalen Klimas in der Arbeiterschaft" um 6 Monate zu verschieben. Währenddessen sollte der längst gekündigte Vertrag weiter in Kraft bleiben. Das spanische Recht sieht diese Möglichkeit vor, sofern nur der leiseste Druck von der Basis ausgeht. Das vertikale Syndikat, in dem die Untemehmer selbst sitzen, hat über den Antrag innerhalb von 15 Tagen zu entscheiden. 15 Tage, die Standard dazu benutzen kann, eine Schwächung der Bewegung abzuwarten, bzw. durch Sanktionen, Entlassungen usw. zu fördern. So war dies ein Versuch, die Bewegung zu spalten, indem es jetzt nur noch um die Alternative ging, entweder den Vorschlag der Firma anzunehmen oder für ein halbes Jahr gar nichts zu erhalten. Währenddessen verlangen die Arbeiter Weiterverhandlung, und zwar auf Basis ihres Entwurfs. Täglich finden Versammlungen und Streiks statt. Diese Absicht des Unternehmens wird werlige Tage später deutlich, als die Verhandlungen am 25.1. fortgesetzt werden. Die „Arbeitervertreter", der "Betriebsrat", hatte inzwischen aufgrund der Drohung des Betriebes den amputierten Entwurf der Arbeiter fallenge. lassen. Der Firmenvorschlag ist jetzt die einzige Verhandlungsbasis.

Am selben Tag schließen sich zu Ende der Arbeitszeit (14.45 Uhr) etwa 1500 Arbeiter in der Kantine einer der beiden großen Fabriken ein, um auf einen ,BR ${ }^{\prime \prime} \mathrm{zu}$ warten, der sie über den Verlauf der Verhandlungen informieren soll, vor allem daüber, auf welcher Grundlage überhaupt verhandelt wird. Der "BR" ignoriert die Anwesenheit der Arbeiter völlig, die bis nach 22 Uhr aushalten. Um 23 Uhr, als die Arbeiter das Haus bereits verlassen haben, wird der Entwurf des Unternehmens ohne Modifikation als neuer Vertrag mit 2-jähriger Laufzeit unterzeichnet und wird damit unanfechtbares Gesetz. Presse und Fernsehen sprechen loch in derselben Nacht von ,wichtigen sozialen und Gehaltsverbesserungen ", um in Klima gegen eventuelle Proteste seitens der Arbeiter zu schaffen.

Als die Arbeiter am nächsten Tag, Samstag, dem 26. 2., vom Abschluß erfah$\mathrm{n}$, reagieren sie mit Vollstreik in beiden großen Fabriken: in Ramirez de Prado Madrid) von Arbeitsbeginn an, in Villaverde (Madrid) ab 10 Uhr. Auf einer Ver- 
sammlung in Ramirez de Prado fordern 2000 Arbeiter

- Ungültigkeitserklärung des Vertrages durch das Arbeitsministerium

- inzwischen Lohnerhöhung unterschiedslos für alle um monatlich 3000 ptas.

- Entiassung des ,Betriebsrates"

- keine Sanktionen gegen die Arbeiter seitens des Unternehmens, wie Lohnentzug, Entlassungen etc.

Am Montag, dem 28.1., setzt sich der Streik in allen Zentren fort, worauf die Betriebsleitung die Polizei ruft, die Ramirez de Prado um 11.30 Uhr und Villaverde um 13 Uhr räumt.

Am Dienstag, 29. 1., räumt die Polizei die zwei übrigen Zentren; damit sitzen etwa 14000 Arbeiter auf der Straße. Ihnen wird zugleich der Lohn für diese Tage entzogen, oft für länger. Das Unternehmen beginnt, die aktivsten Arbeiter zu entlassen. An diesem Tag 28. Elf von ihnen sind Arbeiter, die übrigen Büroangestellte oder Techniker. Acht von ihnen sind ak tive Syndikatsvertreter, einer wurde verhaftet. Die erste Verhaftung.

Am Donnerstag, dem 31. 1., werden Villaverde und Ramirez de Prado wieder geöffnet. In Viliaverde halten die Arbeiter eine Versammlung ab und beschließen, mit den folgenden Zielen weiterzukämpfen:

- Wiedereinstellung der Entlassenen, Aufhebung der Sanktionen (Lohnkürzun. gen etc.), Freiheit für verhaftete Arbeiter

- 500 ptas. täglicher Mindestlohn für den Hilfsarbeiter, 5000 ptas. monatliche Erhöhung für alle Kategorien

- Entlassung der „Betriebsräte“, die den Vertrag unterzeichnet haben ( 6 von ihnen haben sich geweigert)

In Villaverde ist der Streik total; es wird ein weiterer Arbeiter entlassen und fünf werden sank tioniert. In Ramirez de Prado werden 24 Arbeiter mit Lohnentzug auf unbestimmte Zeit bestraft, und es wird gegen sie ein verschärftes Entlassungsverfah. ren eingeleitet. Das bedeutet, daß sie im Sektor Metall in der ganzen Provinz keine Anstellung mehr finden. In der Abteilung Installation in Barcelona das gleiche gegen zehn Arbeiter, sowie in Madrid gegen einen ,BR", der den Vertrag nicht unterzeichnete.

Die Repression in diesen Tagen wird immer stärker. Im Vorort Villaverde beherrscht die Guardia Civil das Straßenbild. Die anderen Zentren haben wir nicht gesehen, aber es sah vermutlich ähnlich aus. Die Kommunikation unter den Streikzentren ist für die Arbeiter schwer aufrechtzuerhalten. Madrid ist erst nach dem Sieg der Faschisten eine große Industriestadt geworden, und das spiegelt sich in der Lage der Industriezentren rund um die Stadt wider. Sie liegen jeweils mehr oder weniger jenseits des Stadtrands ohne Kommunikationswege untereinander, wie Straßen, Busse, Metro, sondern sind nur über das Stadtzentrum verbunden. Die Industrieregionen sind daher leicht voneinander zu isolieren. Die Ausgangsbasis für eine Vereinheitlichung der Kämpfe in den verschiedenen Industrievororten ist hier schlechter als in den organischeren Industrieregionen wie Barcelona oder Bilbao.

Die Zusammenarbeit von Standard, Polizei und Syndikat erweist sich als sehr eng. Auf Veranlassung des Unternehmens beginnt die Polizei, bekannte Arbeiter nachts in ihren Häusern zu verhaften, wenn sie sie tagsüber nicht erwischen kann 
oder die Solidarisierungsgefahr zu groß ist.

Die Streikbewegung ist sehr geschlossen, und die Unterstützung der comisiones obreras fast vollständig. Die comisiones setzen sich aus Nichtorganisierten und Mitgliedern verschiedener Organisationen (darunter die KP, Bandera Roja, O.R.T., sowie katholische Gruppen) zusammen. Sie sind teilweise identisch mit den Anti-1001-comisiones, die im letzten Jahr zur Unterstützung der zehn angeklagten Arbeiterführer (der Prozess Nr. 1001 am 20. 12. 1973) gebildet worden waren und sich über ganz Madrid verteilten. Die comisiones bei Standard agieren weitgehend einheitlich und drücken die Beschlüsse der Versammlungen aus. Der Zusammenhang von Massenbewegung und comisiones obreras ist sehr eng und direkt.

Die wichtigste Aufgabe der comisiones obreras besteht in der Initiierung von gemeinsamen Aktionen und vor allem in deren Koordinierung, sowie Verbreitung von Informationen nicht nur für die Belegschaft, sondern für die ganze Stadt, der Einberufung von Versammlungen usw. Hauptsächlich also organisatorische Aufgaben, und es zeigt sich, daß für eine „Anleitung" des Kampfes, wie sie die außerhalb der comisiones handelnde KP(ML) versucht, keine Notwendigkeit besteht. Die comisiones halten sich an die Beschlüsse der Belegschaftsversammlungen und versuchen, diese durchzuführen, soweit es die Repression zuläßt. Sie stellen den organisatorischen Kern der kämpfenden Arbeiter dar und sind so etwas wie eine ,ausführende Avantgarde" der Bewegung.

Während der Kämpfe selbst leistet es sich außer der ML keine Organisation, einen Führungsanspruch aufzustellen. Große Teile der Arbeiter und sogar der Mitglieder der comisiones können oft keine genauen Angaben über die Organisationszugehörigkeit anderer Mitglieder machen. Das hohe Bewußtsein von der notwendigen Einheit der Bewegung ist vielleicht der wichtigste Charakterzug des gegenwärtigen Stands der spanischen Arbeiterbewegung.

Die höheren Angestellten haben sich dem Kampf größtenteils angeschlossen. Das Personal des Forschungs- und Rechenzentrums scheint dabei eine sehr wichtige Rolle zu spielen: Da dieses Zentrum von allen die längste Zeit im Streik geschlossen war, haben viele dort Beschäftigte Koordinationsaufgaben übernehmen liönnen. So ist z. B. ein „Komitee zur Ausdehnung des Kampfes" entstanden, welches sich um die Erklärung des Kampfes in anderen Betrieben, den Universitäten usw. bemüt, sowie ein „Komitee zur Unterstützung der Entlassenen“, welches die Spenden aus den Unis und Betrieben verteilt. Obwohl die Presse den Kampf weitgehend totschweigt, bzw. immer wieder von der Normalisierung des Arbeitsablaufs redet, ist es gelungen, zumindest in Madrid eine breite Solidarisierung herzustellen. Dennoch ist der finanzielle Druck eine der wichtigsten Waffen in den Händen đes Unternehmens. In einem Land, in dem Streiks verboten sind, gibt es natürlich keine Streikkassen. Das ist die Hauptursache für das Zusammenbrechen vieler Streiks. Bei einem multinationalen Konzern wie ITT allerdings ist die internationale Solidarität der Arbeiter leichter herzustellen als bei anderen Unternehmen. Relativ schnell treffen Spenden aus ITT-Produktionsstätten ein, sowie von größeren Konzentrationen spanischer Arbeitsemigranten im Ausland, so u. a. aus Brüssel, Rom und verschiedenen Orten Frankreichs. 


\section{Der Kampf verschärft sich weiter}

Am Freitag, dem 1. 2., wird in Ramirez de Prado teilweise gestreikt, in Villaverde voll. Mittags wird hier ein Brief von der Betriebsleitung verlesen, der die Entlassung aller streikenden Arbeiter androht und eine halbe Stunde Zeit gibt, zur Arbeit zurückzukehren. Es entsteht eine enorme Spannung und große Gereiztheit im Betrieb. Es finden Auseinandersetzungen innerhalb der Belegschaft statt. Ein als Spitzel bekannter Beschäftigter wird krankenhausreif geschlagen. Zu Betriebssciluß halten die Arbeiter eine Versammlung ab, auf der die Fortsetzung des Kampfes beschlossen wird. Währenddessen werden drei Arbeiter zu Hause verhaftet. Sie können bis zu $72 \mathrm{Std}$. festgehalten werden, ohne daß Anklage erhoben wird. Hat die Polizei bis dahin irgend etwas in Händen, was die Verhaftung begründen könnte, kann sie für die Freilassung eine Kaution verlangen, meist $100000-200000$ ptas. (5-10 000 DM). In der Regel können die Arbeiter die Summe nicht aufbringen. Seit kurzem gibt es die juristische Möglichkeit, verhafte te Arbeiter wegen ihres Nichterscheinens am Arbeitsplatz zu entlassen.

Am Samstag, dem 2. 2., führen die Arbeiter von Villaverde wieder eine Versammlung durch und wiederholen ihre Forderungen. Mittags wird die Fabrik von neuem durch die Polizei geräumt und bleibt nach polizeilicher Verfügung ,auf unbestimmte Zeit" geschlossen. Das Forschungs- und Rechenzentrum ist noch immer geschlossen. Die Zahl der Entlassenen ist inzwischen auf etwa 70 angestiegen, und es finden in Villaverde drei weitere Verhaftungen statt.

Am Montag, dem 4.2., bleiben das Forschungszentrum und Villaverde geschlossen. Standard erklärt die Auflösung der Arbeitsverträge ,des Personals, welches an undisziplinierter Haltung festhielt". Damit sind über 4000 Arbeiter gekündigt, fast die gesamte Belegschaft von Villaverde. Aus Brüssel reist die ITT-Direktion für Europa an, und es tauchen Gerüchte über die Entlassungen einiger Mitglieder der spanischen Unternehmensleitung auf.

Zwei Tage später, am 6. 2., kündigt die Firma individuelle Wiedereinstellung fast aller vorheriger Mitarbeiter an. Zugleich setzt sie die Wiedereröffnung des Werkes Villaverde auf den 8.2. fest. Inzwischen sind schon 10 Syndikatsvertreter und zwei Mitglieder des ,BR "B entlassen, darunter zwei von den sechs Nichtunterzeichnern des Kollek tivabkommens.

7. 2.: Vom Syndikat ist durch die Presse zu erfahren, daß es sich bei den Wiedereinstellungen nur um 3900 Personen handeln soll. Bis jetzt (Anfang Mai) ist das Einstellungsverfahren noch nicht abgeschlossen. Über die Zahl der Entlassenen ist daher nichts Genaues zu sagen. Gegenwärtig sind es noch 17 Personen, von denen der größte Teil einem bestimmten Entlassungsverfahren unterliegt, das die Möglichkeiten, im Metallsektor der Provinz Madrid wieder Arbeit zu finden, sehr verringert. Mehrere Hundert Arbeiter versuchen, mit Beamten des Metallsyndikats im Syndikatsgebäude zu reden. Der zuständige Beamte verschwand, sein Sekretär erklärte die Versammlung für illegal, die Arbeiter hätten ihre Wünsche in schriftlicher Form einzureichen, deren Weiterleitung er ihnen anbot. Einige Meter vom Syndikatshaus in der Madrider Innenstadt griff die Polizei ein und trieb die Arbeiter auseinander. Trotz der Ankündigung der Wiedereröffnung von Villaverde am 8.2. 
läß Standard bereits am 7. 2 einige Hundert Arbeiter hinein. Jeder hat einen Vertrag zu unterschreiben, womit die bisherigen Altersvergünstigungen, besondere Prämien, persönlich erworbene Lohnanteile usw. wegfallen. Alle Arbeiter erhalten durchweg den Status von Neuanfängern. Die Politik des Konzerns ist damit vollends deutlich geworden: durch die Entlassungen ist er einerseits die aktiven Arbeiter los, andererseits fällt das gesamte Sonderzulagensystem fort, das wegen der niedrigen vertraglichen Lohnabsicherung einen relativ hohen Anteil ausmachte.

An Freitag, dem 8. 2., arbeiten angeblich bereits 1700 Beschäftigte. Sie werden in Gruppen zu je etwa 300 Personen ins Fabrikgelände gelassen. Das Gelände wird stark von Guardia Civil bewacht. Es ist kein Wunder, daß die Zeitungen am 9. 2. von voller Normalität des Arbeitsablaufs in Villaverde sprechen können. Für den Samstag, 9. 2., werden weitere 870 Wiedereingestellte erwartet.

Am Morgen des 9. 2. werden zwei weitere Arbeiter vor dem Syndikatsgebäude verhaftet, in dem sie aus Solidarität am Schlichtungsverfahren zwischen Standard und den Entlassenen beiwohnen wollten. Das Verfahren ist gescheitert. Die Arbeiter haben ihre Beschwerde in kollektiver Form eingereicht. Standard erkennt die Kollektivsituation aller Arbeiter im Arbeitskonflikt nicht an und verlangt die Individualisierung der Beschwerden. Dabei hat Standard natülich das ,Recht ${ }^{66}$ auf seiner Seite.

Am Wochenende des 9./10.2. herrscht große Angst unter den Arbeitern vor Lohnentzug, Arbeitsplatzverlust und Verhaftung. Bisher sind zehn Kollegen verlsaftet worden. Obgleich Geldsammlungen eintreffen, ist die finanzielle Lage der Arbeiter sehr schwierig. Ein großer Teil (über $90 \%$ ) hat Lohnverluste zu erleiden als Form von Bestrafungen des Betriebes; die Arbeiter von Villaverde zusätzlich noch in Form von Verlust ihrer Altersanspriiche und Prämien. Für sie bringt der neue Vertrag eine erhebliche materielle Verschlechterung, und das bei einer von der Regie rung angege benen Preissteige rungsrate von 14,2\%.

Am nächsten Tag war die Unruhe in den Produktionsstätten noch groß, aber es fanden keine Kampfaktionen mehr statt. Nicht mehr Angst, sondern Empörung begann wieder sich auszudrücken. Das war die einzige noch mögliche Form des Protestes. Die Polizei hatte auf jeder Etage der Produktionszentren Kontingente postiert, die darüber zu wachen hatten, daß sich keine Ansammlungen von mehr als zwei Personen bildeten. Die Bereitschaft zum Kampf ist nicht gebrochen, aber die Arbeiter haben gegenwärtig keine Möglichkeit mehr. In diesem Sinne ist dieser Arbeitskonflikt für spanische Verhältnisse nicht außergewöhnlich. Arbeitskämpfe brechen meistens nicht aufgrund mangelnden Kampfwillens zusammen, sondern aufgrund der außerordentlich harten Repression, sowie wegen fehlender finanzieller Unterstützung. Die materielle Unterstützung ist daher für die spanische Arbeiterklasse von größerer Bedeutung als in anderen Ländern. Die Entlassenen sitzen fast immer ohne Unterstützung auf der Straße. Die comisiones obreras rufen daher dringend zu Solidarität auf. 
Auch nach der Art des Verlaufes ist der dargestellte Arbeitskonflikt eher typisch als außergewöhnlich. Die Strategie des Kapitals ist in den meisten Fällen ähnlich: entweder das Syndikat für seine Zwecke zu benutzen, wie im geschilderten Konflikt, oder, sofern das Probleme mit sich bringt, direkt auf die staatliche Intervention in die Lohnfestsetzung zu bauen. Die staatliche Repression in Form von Polizei, Militär und Justiz ist sowieso immer unausgesprochenes Druckmittel bei den ,Verhandlungen ${ }^{6}$.

Angesichts dieser Repression ist eine die verschiedenen politischen Gruppen zusammenfassende Einheitsbewegung notwendig. Dieses Sammelbecken der Arbeiterbewegung stellen die comisiones obreras (Arbeiterkommissionen) dar. Daneben gibt es eine Menge geheimer Organisationen von Parteien über Aufbauorganisationen bis hin zu geheimen Gewerkschaften. Aber fast alle Gruppen mit Ausnahme deı ML und der Sozialdemokraten unterstützen die comisiones und arbeiten in ihnen. Die Mls denunzieren ,die comisiones" als von der KP gelenkt, daher revisionistisch (18), die Sozialdemokraten werben fuir ihr eigenes geheimes Syndikat, die UGT, die während des Bürgerkrieges neben den Anarchisten die zweite proletarische Massenorganisation war. Heute ist sie noch vorwiegend in Asturien stark.

Die comisiones obreras sind gegenwärtig die am meisten verbreitete Organisationsform der spanischen Arbeiterbewegung. Dabei ist die Bezeichnung „Organisationsform" nicht ganz richtig, sofern darunter eine feste Organisation verstanden wird. Das wird aus der Geschichte der comisiones deutlich:

Sie sind Ende der fünfziger/Anfang der sechziger Jahre entstanden und fanden zum ersten Male weite Verbreitung während des asturischen Bergarbeiterstreiks im Jahre 1962. Bis dahin gab es in Spanien ausschließlich geheime Organisationen, die wegen ihres Geheimcharakters kaum Massenwirksamkeit erlangten. Von den Organisationen, die sich nach Entstehung der comisiones obreras auf diese neue Organisationsform umstellten, war die KP die erste. Sie löste ihr geheimes Syndikat zugunsten der comisiones auf. Diese neue Bewegung war ungleich demokratischer strukturiert als alle vorherigen Organisationsversuche. Die comisiones entstanden spontan aus der Bewegung und drückten daher am direktesten die politischen Bedürfnisse der Arbeiter aus. Zunächst stellten sie nichts anderes als gewählte Delegierte für die Verhandlungen mit der Kapitalseite dar. Mit dem Abebben des Kampfes verschwanden auch die comisiones. In den folgenden Jahren verbreiteten sich die comisiones über ganz Spanien und wurden mehr und mehr zu ständigen Organen, die über Kampfpausen hinaus existierten.

In dem Maße, in dem die comisiones unabhängig vom Niveau der Einzelkämpfe wurden, entstand die Gefahr ihrer Ablösung von der Bewegung. Die Kritik der Bürokratisierung mag daher in vielen Einzelfällen berechtigt sein, sowie er in vielen anderen Fällen nicht stimmt. Es gibt Betriebe, in denen versucht wird, Gegencomisiones zu schaffen. Dennoch ist ein pauschaler Vorwurf falsch, "die" comisiones seien bürokratisiert oder revistionistisch. Die comisiones befinden sich immer in der

18) vgl. für die pauschalisierende Form der Kritik den Artikel von Jaime Martin, in: Le Monde Diplomatique, Paris Februar 1974, S. 7 
Ambivalenz, direkter Ausdruck einer Massenbewegung zu sein und zugleich mit ihrer Institutionalisierung den Keim einer Ablösung von den Kämpfen in sich zu tragen. Entscheidenden Einfluß auf das Verhalten der comisiones haben in jedem Fall das Niveau und die Kampferfahrung der betreffenden Arbeiter.

Diese Ambivalenz ist auch durch die Repression bedingt. Die Verselbständigung von der Basis geschieht meist unter dem legitimatorischen Hinweis, daß die comisiones nicht offen auftreten dürfen, ohne ihre Existenz zu riskieren. Die Grenzen zwischen comisiones und geheimen Kadern sind besonders in kampfschwachen Perioden und bei verstärkter Repression schwer zu ziehen.

Durch die von vornherein bestehende Einheit von politischem und ökonomischem Kampf wird die Trennung der Arbeiterbewegung in Gewerkschaften und Partei tendenziell überwunden. Zwar ist die KP sowohl in den comisiones als auch als Partei die stärkste politische Richtung in Spanien, aber ihre Existenznotwendigkeit als Organisierungsinstanz für einen besonderen politischen Kampf im Gegensatz zum ökonomischen, den sie den comisiones zuweist, ist durch die von ihr einst wesentlich geförderte Dynamik der comisiones bedroht. Santiago Carrillo, der Generalsekretär der KP, sieht diese Gefahr auch sehr klar (19). Diese Entwicklung wird sich in den nächsten Jahren sicherlich fortsetzen.

Das Jahr 1974 hat mit dem geschilderten Konflikt bei Standard Electrica begonnen. Die meisten der zweijährigen Kollektivabkommen der kampfstärksten Unternehmen müssen in diesem Jahr emeuert werden, dann wieder 1976, wenn nicht bis dann die Durchsetzung kürzerer Laufzeiten gelungen ist. Durch die Politik der EG.Länder, die Zahl der Arbeitsemigranten beizubehalten oder sogar zu verringern, hat Spanien 1974 evtl. eine Arbeitslosenzahl von über einer Million zu gewärtigen. Ein wichtiges Element spanischer Beschäftigungspolitik war stets die Emigration von Arbeitskräften. Allein 1972 waren es über 100000 (20). Das Regime plant, für 1974200000 neue Arbeitsplätze zu schaffen. Nach den bisherigen Erfahrungen ist es unwahrscheinlich, daß man diese Z Zahl erreichen wird.

Wenn aufgrund der großen Arbeitslosigkeit in diesem Jahr sich die Kämpfe evtl. nicht ausweiten, so werden sie sicherlich in den nächsten Jahren wieder einen Aufschwung erfahren, wenn sich die konjunkturelle Lage in Spanien und Westeuropa verbessert.

Das Regime scheint dieser Situation nicht gewachsen zu sein, was sich auch in der Arbeitsgesetzgebung der letzten Jahre zeigt: Weder das Syndikatsgesetz von 1971, noch die Erneuerung des Gesetzes über Kollektivabkommen vom Dezember 1973 bringen wirkliche Veränderungen. Schon seit Jahren wird ein Gesetz über Kollektivkonflikte hin ausgezögert. Das spanische Regime verfügt über keinerlei integrative Kraft. Stattdessen steigert sich die Repression, was sich z. B. in der einem Ausnahmezustand ähnlichen Situation in den vier kampfstarken baskischen Provinzen seit Dezember 1973 ausdrückt. Diese Tendenz wird zunehmen. Die Richtung

19) „,... wir sehen in unseren Reihen das Aufkommen des, ,Syndikalisten“, der den Arbeitskampf vor alles andere stellt. Ohne dieses Risiko zu übertreiben, müssen wir aufpassen, daß es sich nicht in eine Gefahr verwandelt, vor allem in der Zukunft." (Rede Santiago Carrillos auf dem VIII. Kongreß der PCE, VIII Congreso del PCE, Bukarest 1972, S. 74

Cruz/Serrano, a. a. O., S. 246 
der spanischen Politik besteht eindeutig in einer verschartten Kepression.

Zugleich schürt das Regime Liberalisierungsillusionen. Die offizielle politische Diskussion kreist seit Monaten um Öffnung des Systems (,aperturismo"), man sucht nach Wegen, den neuerdings als reif bezeichneten Bürger in die Entscheidung über den politischen Prozeß in Spanien einzubeziehen. In den Cortes, dem Ständeparlament, Zulassung politischer Organisationen debattiert, die freilich die Prinzipien des ,Movimiento", der falangistischen Eirheitsbewegung anzuerkennen haben. Doch bereits diese "Liberalisierung" scheint das Regime zu fürchten. Es preist sich als volksverbundener als die vorherigen an, da es die Bürgermeister der Städte vom Volk wählen lassen will, die bisher von oben eingesetzt wurden. Deren Machtbefug. nis erstreckt sich allerdings auch nur vom Straßenbau bis zum Kampf gegen die städtische Luftverschmutzung. Anläßlich deren Wahl wird sich für den ,,reifen Bürger" an der Unne noch nicht die große gesellschaftliche Alternative anzeigen.

Soweit die spektakulärsten aktuellen Projekte des Regimes, welche von der ausländischen Presse häufig als vielversprechende Signale mißverstanden werden. Diese Initiativen enthalten durchweg keine, die Lebenssituation der lohnabhängigen Bevölkerung verbesse mde Substanz. Die anfangs gestelite Frage, wieweit es in Spanien überhaupt eine ,Liberalisierung“ gebe, läßt sich für die Lohnabhängigen negativ beantworten. Anders für das Kapital. Es hat mit dem politischen Wechsel von 1958 durchaus eine „Liberalisierung" des Kapitalverkehrs gegeben. Das ausländische Kapital ist in großem Umfang nach Spanien eingeströmt und hat weite Möglichkeiten der Gewinntransferierung garantiert bekommen. Die vormals diese Kapitale hemmenden Vorschriften sind entweder aufgehoben oder zumindest stark durchlöchert worden. Spanien ist für ausländische Investitionen ein sehr attraktives Land. Die Löhne sind niedrig und die Streikziffern sind mit 1973 über 12 Mio. ausgefallenen Arbeitsstunden noch relativ gering (verglichen etwa mit Italien). Spanien wird zunehmend zu einem Anziehungsland für Kapital aus arbeitsintensiven Sektoren, welche Produktionsstätten im Umkreis des EG-Marktes sucht. Gerade diese arbeitsintensiven Sektoren sind aber meistens zugleich die konflik tivsten. Um diese Anziehungskraft zu erhalten und gleichzeitig durch ausländische Investitionen die Arbeitslosigkeit zu mildern, wird ein wesentliches Ziel auch der neuen Regierung die Repression sein.

\section{Aufruf}

Inzwischen sind bei der comision obrera von Standard Electrica mehr Geldspenden eingetroffen, als für die Unterstïtzung der entlassenen Arbeiter und ihrer Familien zur Zeit benötigt werden. Die intemationale Solidarität stellte sich vorwiegend im Rahmen des ITT-Konzerns her. Der größte Teil der ausländischen Spenden kam aus ITT-Niederlassungen. Aber auch Organisationen spanischer Arbeitsemigranten schickten beträchtliche Summen zur Unterstützung ihrer Kollegen im Heimatland. Durch die Möglichkeit dieser Formen der Solidarität erhalten die multinationalen Konzerne in Spanien eine wichtige neue Funktion für die Arbeiterbewegung. Sie werden zu den finanziellen Unterstützungszentren für eine Stadt oder Provinz im 
Verlauf von Arbeitskämpfen.

Das ist auch gegenwärtig in Madrid der Fall. Die comision von Standard verteilt das bei ihr eingegangene Geld an andere Betriebe, die gerade einen Arbeitskampf führen. Ende April befanden sich in Madrid drei Mittelbetriebe im Arbeitskampf und ein Unternehmen hatte die gesamte Belegschaft von etwa 500 Kollegen entlassen. Diesen kleineren Betrieben ist eine Mobilisierung der Öffentlichkeit viel schwerer möglich als ITT. Die materielle Unterstützung ist vor allem während des Arbeitskampfes wichtig, nicht nur nach seiner Beendigung, wenn die entlassenen Arbeiter noch stärker auf die Solidarität ihrer Kollegen angewiesen sind.

Es ist von der Zeitschrift "links" ein zweckgebundenes Sonderkonto eingerichter worden, und es wird zu Spenden aufgerufen:

Sonderkonto ,Spanien",

Hannelore Vack,

Nr. $1749961 / 11$

bei der Bank für Gemeinwirtschaft

Niederlassung Offenbach

Soeben erschienen!

Über die Bedeutung des neuen Lohnrahmentarifvertrages in der Metallindustrie von Nordwürttemberg-Nordbaden

Herausgegeben vom Arbeitskollektiv LRTV und MTV in Stuttgart

Selbstverlag. 44 Seiten. DM 2,90 Bezug: Politladen 852 Erlangen, Postfach.

\section{PAPIERSCHEICHS}

Nach der Öl- jetzt die Papierkrise! Wir bitten, auch im Namen zahlreicher anderer linker Verlage, die Käufer und Leser unserer Bücher und Zeitschriften folgendes zu beachten:

Seit Jahresbeginn sind die Preise für Filme, Farben, Druckhilfsstoffe stärker angestiegen als bisher, am heftigsten trifft uns jedoch die Lage auf dem Papiermarkt: der Preis für das Papier, auf dem z. B. die "Probleme des Klassenkampfs" gedruckt werden, ist vom Spätherbst 1974 bis einschließlich der Anfang Juli 1974 terminierten Preiserhöhung um $75 \%$ gestiegen! Gleichzeitig sind die Preise für Umschlagmaterial um mehr als $50 \%$ raufgegangen. Das Ausweichen auf billigere Papiersorten wird gleichzeitig enorm erschwert, da akzeptable Alternativsorten bis zu 5 Monaten Lieferzeit haben. Zusammen mit Preiserhöhungen in anderen Sektoren (Postgebühren, Elektrizität, Bindekosten, Kreditkosten) ist die Situation leider die, daß auch weitere Erhöhungen der Endpreise unserer Titel in Zukunft nicht zu vermeiden sind.

Politladen - Verlag und Druckerei 